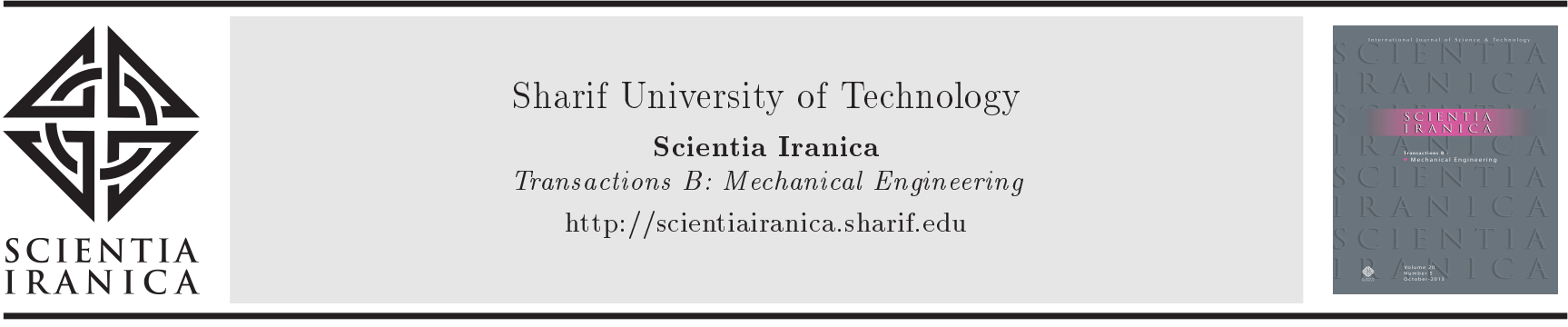

\title{
Mathematical modeling of thermal contact resistance for different curvature contacting geometries using a robust approach
}

\author{
M.H. Shojaeefard* and K. Tafazzoli Aghvami \\ School of Mechanical Engineering, Iran University of Science and Technology, Tehran, P.O. Box 16765-163, Iran.
}

Received 17 April 2018; received in revised form 14 May 2018; accepted 7 July 2018

\author{
KEYWORDS \\ Transient simulation; \\ Thermal contact \\ resistance; \\ Thermal contact \\ conductance; \\ Artificial neural \\ network modeling; \\ Surface interaction.
}

\begin{abstract}
Nowadays, researchers have become interested in acquiring deeper knowledge concerning Thermal Contact Conductance (TCC) and Thermal Contact Resistance (TCR) existing among various types of metals during heat transfer occurrence in the nuclear reactor, thermal control system of spacecraft, and heat exchangers. In the present study, Artificial Neural Network (ANN) coupled with Multi-Layer Perceptron (MLP) modeling was utilized to predict transient temperature contour on various contacting surfaces such as flat-flat, flat-cylinder, and cylinder-cylinder. In order to develop an accurate transient model, the parameters of metals including position, time, and roughness were used as input parameters, and temperatures of solid bodies were selected as the target parameter of the model. Modeling results demonstrate that ANN-based modeling outperforms other numerical methods in terms of accuracy. Moreover, values of Average Absolute Relative Deviation (AARD) and coefficient of determination $\left(R^{2}\right)$ for the overall data are 0.056 and 0.996 , respectively, which prove the accuracy and robustness of the proposed model.

(C) 2019 Sharif University of Technology. All rights reserved.
\end{abstract}

\section{Introduction}

Heat transfer is a key issue for metallic bodies in contact for designing heat exchangers, nuclear reactor cooling, control systems for spacecraft, and microelectronics cooling. The most important parameters affecting heat transfer across the interface include contact loading, surface roughness, and thermophysical and mechanical properties. When two solid bodies are in touch, their physical contacts are limited to the finite number of separated points at their interface [1]. The real contact area is seen on a microscopic scale, which is

*. Corresponding author. Tel.: +9821 77240540; Fax: +9821 r7240488

E-mail address: mhshf@iust.ac.ir (M.H. Shojaeefard)

doi: 10.24200 /sci. 2018.50771 .1856 relatively very small compared to the apparent contact area. Macro-contact is created due to the surface curvature of the contacting bodies. The relatively high temperature difference occurs between the interfaces, because following the flow of heat through the macro contact, it must pass through the micro-contacts to conduct from one surface to another [2]. A constriction on the contact surface in heat transfer is created by this phenomenon called Thermal Contact Resistance (TCR) and is defined as follows $[1,2]$ :

$$
R_{c}=\frac{\Delta T}{q},
$$

where $\Delta T$ denotes the temperature drop between two contacting surfaces, and $q$ is the heat flux, defined as follows [2]: 


$$
q=\frac{d}{d A}\left(\frac{d Q}{d t}\right)
$$

where $Q$ and $t$ are heat transfer between surfaces and time, respectively.

There are experimental, analytical, and numerical models developed for TCR prediction; however, these models are not general and are suitable for specific cases [2].

It was found that several parameters such as the type of contaminant or lubricant, temperature and interfacial pressure, the geometry of contacting surfaces on both micro and macro scales, and the type of contacting materials were the most important factors. Thermal contact resistance can be measured, while the system is in the steady state or transient condition [2$4]$.

Clausing and Chao (1965) proposed a mode for thermal contact resistance in a vacuum environment. Their results showed that the macroscopic constriction is the dominant parameter. They studied the impact of material properties, the degree of conformity of mating surfaces under load, surface films, surface roughness, creep, additional interstitial material, and mean interface temperature. The model predicted the thermal contact conductance quite well [5]. The assumption for most of the prediction models of the thermal contact resistance is flat surface due to its simplicity. Marotta et al. (2001) developed a thermomechanical model including both microscopic and macroscopic thermal resistances for non-flat roughened surfaces with non-metallic coatings. Their model forecasts the thermal contact resistance of several non-metallic coatings deposited on metallic aluminum substrates satisfactorily [6]. Mikic and Rohsenow (1966) proposed a theoretical model to predict the conductance of cylinders and spheres [7]. Thomas and Sayles (1975) studied the relationship among roughness, flatness deviation, and contact resistance [8]. A new method for solving this problem is the inverse problem method. However, authors believe that modeling based on artificial intelligence is more accurate than the previously reported models in the literature.

In this study, Artificial Neural Network (ANN) is used to predict the thermal contact resistance on contacting surfaces for the cylinder-cylinder, cylinderflat, and flat-flat contact surfaces in the transient condition. The contacting surface is an alloy made of brass. The length of all specimens is $30 \mathrm{~mm}$ with a diameter of $25 \mathrm{~mm}$. Each specimen has four holes with a depth of $12.5 \mathrm{~mm}$ and a diameter of $0.8 \mathrm{~mm}$. The diameter of all curved surfaces is $25 \mathrm{~mm}$ (see Figure 1).

\section{Data collection}

This investigation is a computational study; therefore, all of the experimental data used in this study were extracted from literature [1,9-11]. Extracted data contain 287 total experimental data that belong to various parameters of roughness, position, and time, resulting in different temperatures. It is clear that parameters of roughness, position, and time are considered as input parameters of the model, and temperature is the target of the predictive modeling.

\section{ANN modeling}

Artificial Neural Network (ANN) has been developed based on human brain. An artificial neural network similar to human brain has many small processing modules (called neurons) that are connected to each other, the same as network. In order to determine how the outputs and inputs of a system are related, an ANN can be applied. An ANN consists of the neurons, the computational blocks that are similar to biological brain cells and are simple units building the related layers and that their computational relations help determine the network performance [12-14]. A set of neurons is organized in a layer, and their outputs are weighed and utilized as inputs for the forward layer. By using trials and errors, the number of layers and brain cells in each layer can be calculated [15-17]. In addition, there exists an activation function (transfer function) in the input, hidden, and output layers for each neuron. These activation functions can have different forms to define the type of ANN. Some of them are presented in the form of Eqs. (3) to (6):

Linear function:

$$
f(x)=x \text {. }
$$

Hyperbolic-tangent sigmoid function:

$$
f(x)=\frac{2}{1+e^{-2 x}}-1 .
$$

Logarithmic sigmoid function:

$$
f(x)=\frac{1}{1+e^{-x}} .
$$

Exponential function:

$$
f(x)=10^{-x} .
$$

\subsection{Error Back Propagation (EBP) learning approach}

In the current investigation, a multi-layer neural network has been employed to model the relationships between the output and input variables. A simple demonstration of this type of network is shown in Figure 2. In the first stage, training, the output of the network is compared with the target value (results of experiments), and the calculated error of the network is back-propagated to the previous layers $[18,19]$. Then, 


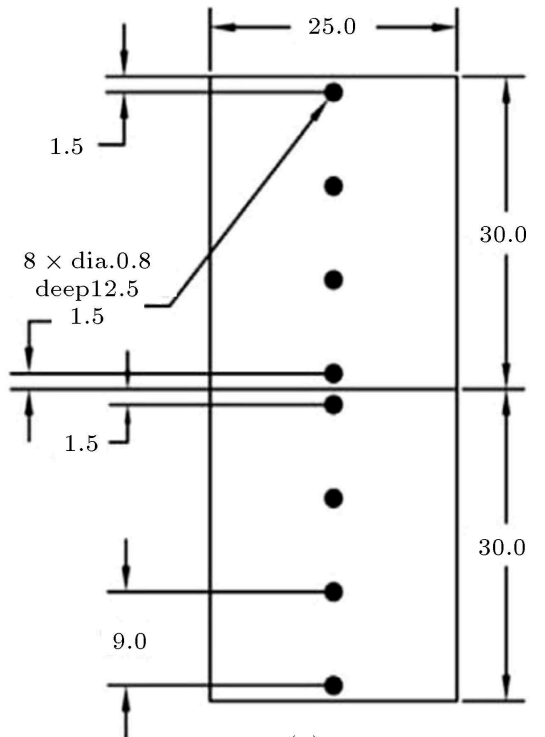

(a)

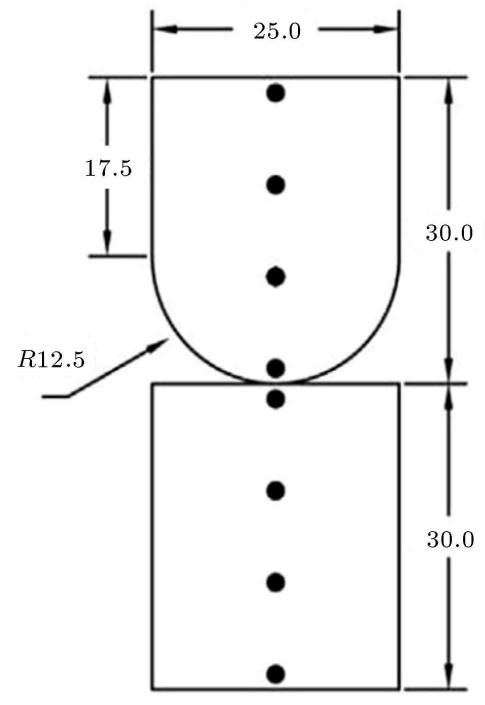

(b)

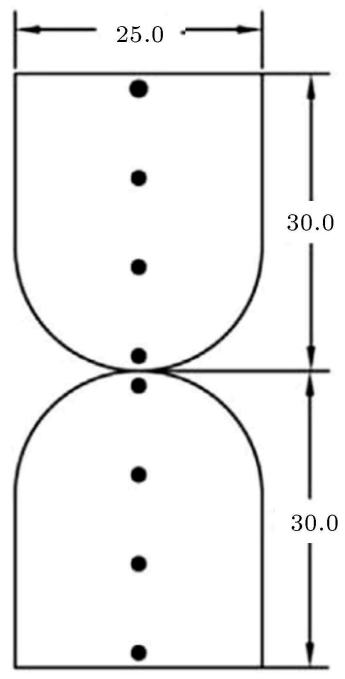

(c)

Figure 1. Schematic of different contact configurations.

the weights will be tuned using these propagated errors. This training approach is called the Error BackPropagation (EBP) [12,20].

To describe how the EBP has been practiced and

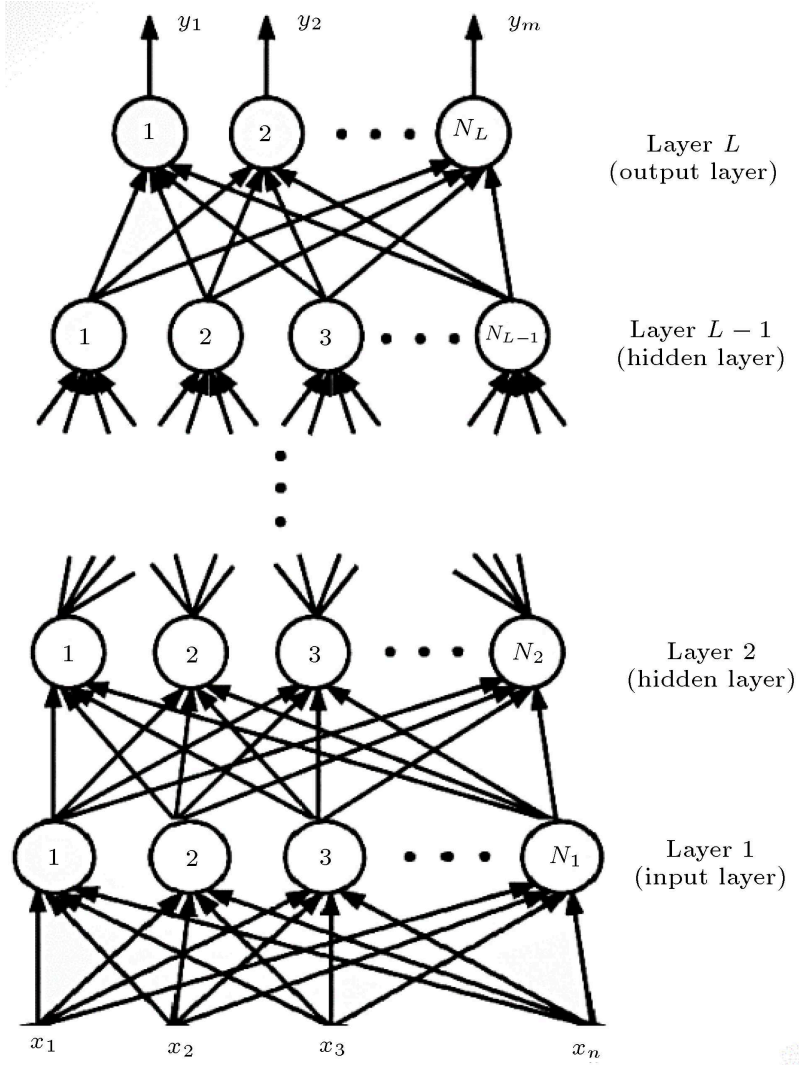

Figure 2. Architecture of an ANN, showing the relationship between the input variables and the output variable. for the sake of simplicity without loss of generality, it has been assumed that the network has only two layers: one input and one output. Each input is multiplied by weights of the input layer neurons $\left(w_{i h}\right)$ and is added to a bias value $\left(b_{h}\right)$ to form the activation $\left(a_{h}\right)$. This statement can be written in the vector form as follows:

$$
a=W^{T} I+B
$$

where $I$ is the input vector, $W$ is the weight matrix of the first layer, $B$ is the vector containing biases of input layer neurons, and $a$ is the vector of each neuron activation. After calculating activations of all input neurons, outputs of all of them are estimated using a transfer function as follows:

$$
O_{h}=\frac{2}{1+e^{-2 a_{h}}}-1,
$$

where $O_{h}$ is the output of input neuron number $h$. These computed values will be weighed and added to biases again to establish the activations of the output layer $\left(a_{o}\right)$. The linear transfer function is applied, and output, $O$, is calculated. Then, the error is computed as the difference between the calculated outputs and their corresponding experimental results known as the target data. This process builds the forward step of the back-propagation scheme, and the estimated errors are back propagated through the network to tune weights. Weights are tuned using generalized delta rule as follows:

$$
W_{\text {new }}=W_{\text {old }}-\eta E O
$$

where $W_{\text {new }}$ is the tuned weight, $W_{\text {old }}$ is the weight before tuning, $\eta$ is the learning rate, usually chosen in 
the range [0 1], and $E$ is the estimated error. Weight tuning will be completed for all connections. Errors for all train data are accumulated, and the algorithm will be run until the terminal conditions (predetermined value) are met. ANN modeling of this research was completed by MATLAB software and ANN toolbox.

\section{Modeling the temperature of surface of contacting surfaces using ANN}

\subsection{Structural selection of $A N N$}

A well-trained multilayer network was utilized to predict temperature $(\mathrm{K})$ as output by considering consuming time $(\mathrm{s})$, position $(\mathrm{cm})$, and roughness parameters as inputs. The Damped Least-Squares (DLS) method was used as the training procedure. Hyperbolic tangent sigmoid (Eq. (4)), logarithmic sigmoid (Eq. (5)), and exponential (Eq. (6)) functions were respectively selected as the activation functions of input, hidden, and output layers for each neuron. The optimum structure in the given network, which has been obtained following the trial-and-error experiment, consists of nine neurons for input layer, one neuron for output, and six and three neurons for two hidden layers, respectively.

Furthermore, based on all 287 sets of experimental data, 26 were picked out to evaluate network ability in forecasting the temperature, and the others are used for the training procedure (approximately 10\%). It should be mentioned that in order to construct a network, an in-house MATLAB code was developed. The weights and biases with respect to each layer are presented in Table 1.

\section{Results and discussion}

In order to evaluate the performance of the selected ANN, Root Mean Squared Error (RMSE) and coefficient of determination $\left(R^{2}\right)$ were applied as criteria, which are expressed below:

$$
\begin{aligned}
& \text { RMSE }=\sqrt{\frac{1}{N} \sum_{i=1}^{N}\left(z_{i}^{\text {act ual }}-z_{i}^{\text {predicted }}\right)^{2}}, \\
& R^{2}=1-\frac{\sum_{i=1}^{N}\left(z_{i}^{\text {actual }}-z_{i}^{\text {predicted }}\right)^{2}}{\sum_{i=1}^{N}\left(z_{i}^{\text {actual }}-\overline{z^{\text {actual }}}\right)^{2}},
\end{aligned}
$$

where $z_{i}^{\text {actual }}$ denotes the $i$ th experimental temperature, which has been extracted from literature, $\overline{z^{\text {actual }}}$ represents the average of actual data, $z_{i}^{\text {predicted }}$ is the forecasted temperature value by the model, and $N$ is the total number of experimental data. It should be noted that authors selected RMSE over common MSE as a criterion to assess the model.

The values of root mean squared error and coefficient of determination for different processes of training and testing and, also, for the overall data are reported in Table 2. Since the reported values for $R^{2}$ and RMSE are close to 1 and 0 , respectively, it can be concluded that the prediction of this model shows a high degree of accuracy; thus, the selected ANN can be trusted. In order to find the most effective parameter in thermal contact conductance and temperature distribution in solid bodies with various contacting surfaces, sensitivity analysis has been implemented. Modeling results reveal that the roughness parameter is the most effective one that has a direct relation with temperature distribution and reverse relation with thermal contact conductance.

A comparison between model output and exper-

Table 2. Statistical parameters.

\begin{tabular}{lcc}
\hline \multicolumn{1}{c}{ Parameters } & AARD & $\boldsymbol{R}^{\mathbf{2}}$ \\
\hline Flat-Flat & 0.042614 & 0.996987394 \\
Flat-Cylinder & 0.075725728 & 0.999848863 \\
Cylinder-Cylinder & 0.054438686 & 0.999926104 \\
Total & 0.056961054 & 0.996762361 \\
\hline
\end{tabular}

Table 1. Weight and bias of layers in the proposed ANN network.

\begin{tabular}{cccccc}
\hline \multicolumn{2}{c}{ Weight to layer $\mathbf{1}$} & Weight to layer 2 & Bias to layer 1 & Bias to layer 2 \\
\hline 4.8024 & -0.81675 & 2.0155 & -0.33084 & -6.3627 & 0.6154 \\
2.4355 & 0.10391 & -0.91506 & 0.50689 & -3.9994 & - \\
-1.6578 & 0.17021 & 3.7496 & -0.15722 & 0.72731 & - \\
0.89536 & 0.12785 & 3.7827 & -0.77351 & -1.3464 & - \\
-1.2227 & 0.079443 & 0.55339 & 0.5787 & -1.6448 & - \\
-3.4585 & 0.020737 & 4.2148 & 0.50509 & -1.4907 & - \\
-0.37401 & 5.5576 & -4.0305 & -0.26484 & -3.4919 & - \\
0.89147 & 4.9334 & -0.36294 & -1.4452 & 5.2269 & - \\
-0.29383 & 5.2732 & -3.5636 & 0.057309 & 3.0969 & \\
\hline
\end{tabular}


imental temperature has been made in the training stage, and the results are presented in Figure 3. As can be observed, the model forecasted the temperature with high precision in this stage. Afterwards, the model

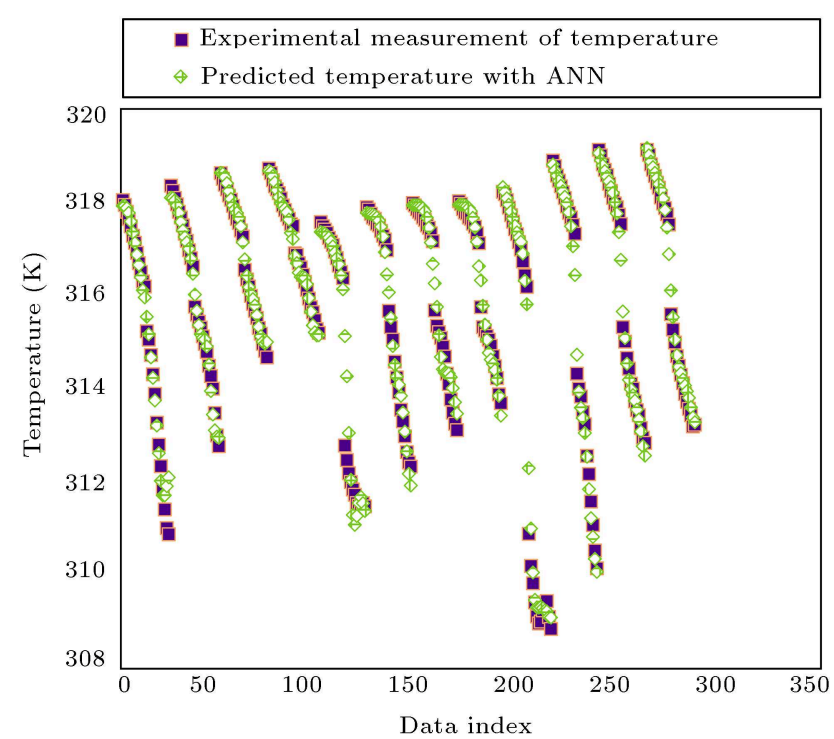

Figure 3. Comparison between experimental and predicted values of temperature along the two species.
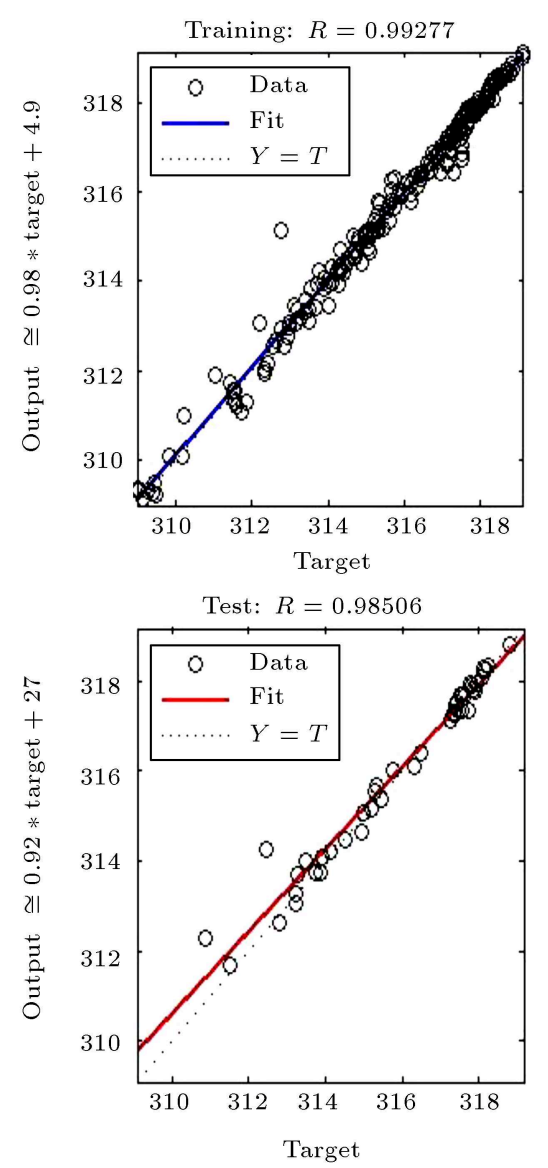

has been evaluated by predicting the test dataset, and a high degree of accuracy has been observed, which concludes the model validation. The results are shown in Figure 4. The predicted values by the neural network model are put on experimental data, as shown in Figure 3 . The predicted values by the ANN-based model are close to $y=x$ line, as shown in Figure 5. Therefore, as shown by the final results of Figure 5, the new neural network model is reliable enough to predict the temperature. Finally, an overall assessment for the relative deviation has been conducted, and the outcomes are presented in Figure 6. Relative deviations of the simulation results from experimental data are bounded between $-1 \%$ to $1 \%$, which is acceptable for ANN-based modeling. According to these figures, the model has shown an excellent ability to estimate the temperature value, and ANN-based modeling is robust for the prediction of temperature on the surface of the two contacting solid bodies with various contacting curvatures.

\section{Conclusion}

An effort has been made for the first time to develop an ANN model to predict temperature distribution and thermal contact conductance between solid bodies
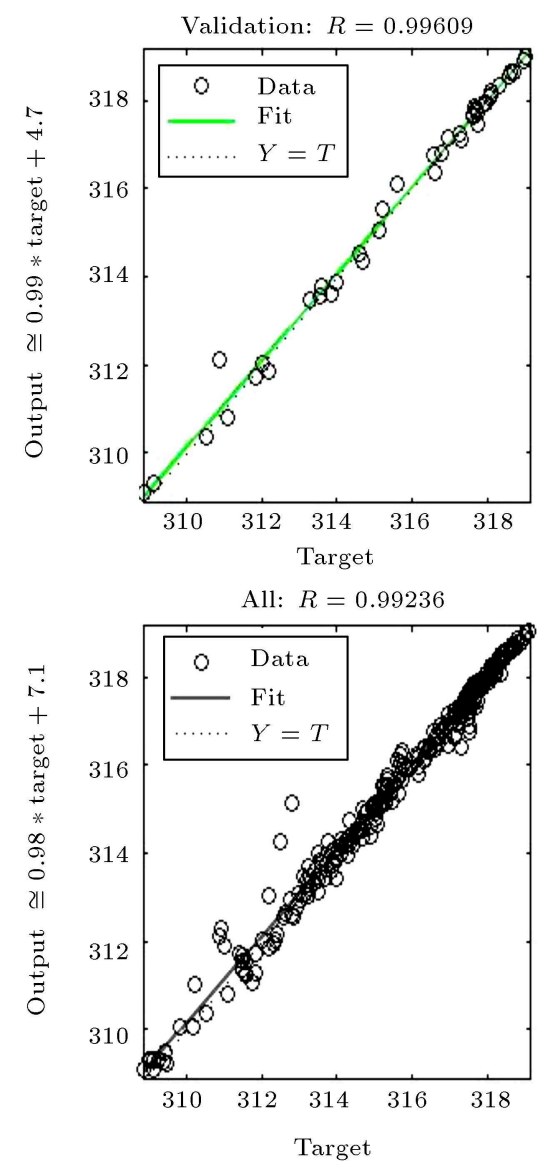

Figure 4. Scatter plot for the training, validation, and testing steps. 


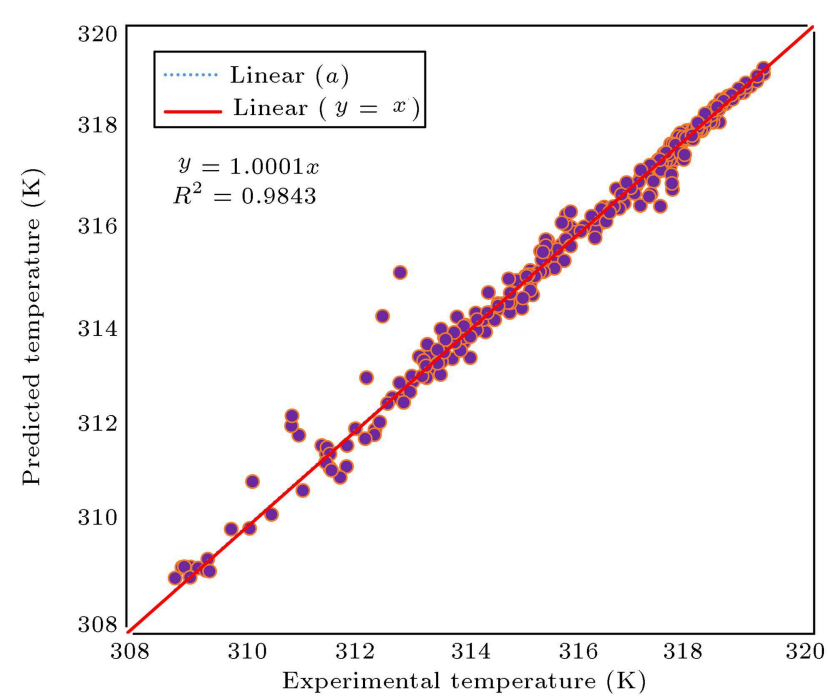

Figure 5. Scatter plot for the experimental versus predicted values of temperature for the two contacting surfaces with various contact curvatures.

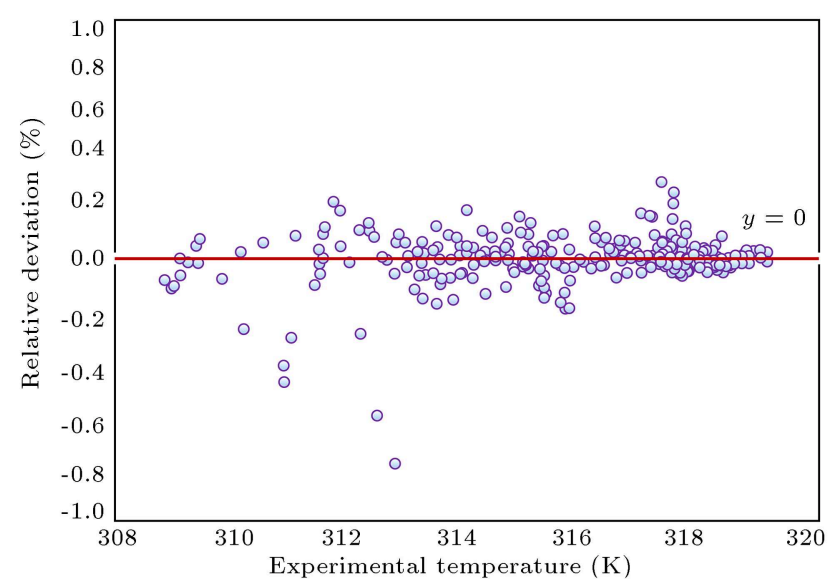

Figure 6. Relative deviation for the predicted values of temperature from experimental data.

with various curvature contacts using four fundamental variables of metal type and environmental conditions in order to facilitate data collection by engineers wherever it is needed such as designing process of heat transfer between contacting surfaces. The developed ANN showed a promising performance in forecasting the temperature distribution of these specific solid bodies in the investigated range of environmental conditions by researchers. This study unveiled that a welltrained, fine structure of the selected ANN has its unique merits such as quick functionality, low cost, and precise prediction ability for the required value. The proposed model was developed for the first time and its acceptable performance was verified.

\section{References}

1. Kumar, S. and Tariq, A. "Steady state experimental investigation of thermal contact conductance between curvilinear contacts using liquid crystal thermography", Int. J. Therm. Sci., 118, pp. 53-68 (2017).

2. Shojaeefard, M.H. and Goudarzi, K. "The numerical estimation of thermal contact resistance in contacting surfaces", Am. J. Appl. Sci., 5(11), pp. 1566-1571 (2008).

3. Wang, S., Xie, T., and Xie, H. "Experimental study of the effects of the thermal contact resistance on the performance of thermoelectric generator", Appl. Therm. Eng., 130, pp. 847-853 (2018).

4. Seok, J., Kim, D., and Kim, S. "Overall thermal conductance and thermal contact resistance in noinsulation REBCO magnet", IEEE Trans. Appl. Supercond., 28(3), pp.1-5 (2018).

5. Clausing, A.M. and Chao, B. "Thermal contact resistance in a vacuum environment", J. Heat Transfer, 87(2), pp. 243-250 (1965).

6. Marotta, E.E., Fletcher, L.S., and Dietz, T.A. "Thermal contact resistance modeling of non-flat, roughened surfaces with non-metallic coatings", J. Heat Transfer, 123(1), pp. 11-23 (2001).

7. Mikic, B. and Rohsenow, W. "Thermal contact resistance", DSR 74542-41, Mech. Eng. Department, MIT (1966).

8. Thomas, T. and Sayles, R. "Random process analysis of effects of waviness on thermal contact resistance", ASME Conf. on Thermophys. Heat Transfer, pp. 674691 (1975).

9. Padilha, R.S. "An analytical method to estimate spatially-varying thermal contact conductance using the reciprocity functional and the integral transform methods: Theory and experimental validation", Int. J. Heat Mass Transfer, 100, pp. 599-607 (2016).

10. Shojaeefard, M.H. and Goudarzi, K. "The numerical estimation of thermal contact resistance in contacting surfaces", Am. J. Appl. Sci., 5(11), pp. 1566-1571 (2008).

11. Prasher, R. "Acoustic mismatch model for thermal contact conductance of van der Waals contacts under static force", Nanoscale and Microscale Thermophys. Eng., 22(1), pp. 1-5 (2018).

12. Hemmat Esfe, M., Wongwises, S., Esfandeh, S., and Alirezaei, A. "Development of a new correlation and post processing of heat transfer coefficient and pressure drop of functionalized $\mathrm{COOH}$ MWCNT nanofluid by artificial neural network", Curr. Nanosci., 14(2), pp. 104-112 (2018).

13. Hemmat Esfe, M., Ahmadi Nadooshan, A., Arshi, A., and Alirezaei, A. "Convective heat transfer and pressure drop of aqua based $\mathrm{TiO} 2$ nanofluids at different diameters of nanoparticles: Data analysis and modeling with artificial neural network", Physica E, 97, pp. 155-161 (2018). 
14. Abdollahi, A. and Shams, M. "Artificial neural network modeling of a deflector in a grooved channel as well as optimization of its effective parameters", Heat Mass Transfer, 54(1), pp. 59-68 (2018).

15. Cook, G.E. "Weld modeling and control using artificial neural networks", IEEE. Trans. Ind. Appl., 31(6), pp. 1484-1491 (1995).

16. Hojjat, M. "Modeling heat transfer of non-Newtonian nanofluids by using hybrid ANN-metaheuristic optimization algorithm", J. Part. Sci. Tech., 12(3), pp. 45-54 (2018).

17. Hemmat Esfe, M., Abbasian Arani, A.A., Shafiei Badi, R., and Rejvani, M. "ANN modeling, cost performance and sensitivity analyzing of thermal conductivity of DWCNT-SiO 2 /EG hybrid nanofluid for higher heat transfer", J. Therm. Anal. Calorim., 131(3), pp. 23812393 (2018).

18. Tafarroj, M.M. "Artificial neural network modeling of nanofluid flow in a microchannel heat sink using experimental data", Int. Commun. Heat Mass Transfer, 86, pp. 25-31 (2017).

19. Ghahdarijani, A.M., Hormozi, F., and Asl, A.H. "Convective heat transfer and pressure drop study on nanofluids in double-walled reactor by developing an optimal multilayer perceptron artificial neural network", Int. Commun. Heat Mass Transfer, 84, pp. 1119 (2017).
20. Rumelhart, D.E., McClelland, J.L., and Group, P.R. "Parallel Distributed The MIT Press, Cambridge, MA (1986). The MIT Press, Cambridge, MA (1986).

\section{Biographies}

Mohammad Hassan Shojaeefard was born in 1950. He received his BSc degree in Mechanical Engineering in 1977 from Iran University of Science and Technology and MSc and PhD degrees in 1984 and 1987, respectively, from the University of Birmingham. He is currently a Professor at the School of Mechanical Engineering, Iran University of Science and Technology. His research interests include experimental and computational fluid mechanics, heat transfer, internal combustion engines, turbomachinery and design of machines, and energy systems.

Kian Tafazzoli Aghvami was born in 1989. He received his BSc and MSc degrees in Mechanical Engineering in 2011 and 2013, respectively, from Iran University of Science and Technology, Tehran, Iran, where he is currently a PhD Candidate at the School of Mechanical Engineering. His research interests include heat transfer, inverse methods, computational fluid dynamics, internal combustion engines, and renewable energy. 